\title{
Publisher Correction: Integrated microthermoelectric coolers with rapid response time and high device reliability
}

Guodong Li (iD, Javier Garcia Fernandez, David Alberto Lara Ramos, Vida Barati, Nicolás Pérez, Ivan Soldatov, Heiko Reith, Gabi Schierning and Kornelius Nielsch

Correction to: Nature Electronics https://doi.org/10.1038/s41928-018-0148-3, published online 12 October 2018.

In the version of this Article originally published, in the Methods section 'Analytical calculation and FEM simulation' owing to a technical error, the first equation was incorrect as:

$$
K_{\text {eff }}=\frac{S_{\text {eff }} \times T_{0}}{\mathrm{c}}
$$

It has now been replaced with the correct equation:

$$
\Delta T=\frac{S_{\text {eff }} \times I \times T_{\mathrm{c}}-\frac{1}{2} I^{2} \times R_{\mathrm{eff}}-Q_{\mathrm{c}}}{K_{\mathrm{eff}}}
$$

In addition, in the section 'Conclusions', the packing density mistakenly read ' 5,000 leg pairs per $\mathrm{cm}^{2}$ ' and has now been corrected to read ' 5,500 leg pairs per $\mathrm{cm}^{2}$ '.

Published online: 5 November 2018

https://doi.org/10.1038/s41928-018-0168-z 